\title{
Mineralocorticoid Receptor-Dependent Proximal Tubule Injury Is Mediated by a Redox-Sensitive mTOR/S6K1 Pathway
}

\author{
Adam T. Whaley-Connell ${ }^{\mathrm{a}-\mathrm{d}, \mathrm{g}} \quad$ Javad Habibi ${ }^{\mathrm{a}-\mathrm{d}, \mathrm{h}} \quad$ Ravi Nistala $^{\mathrm{a}-\mathrm{d}, \mathrm{g}}$ \\ Vincent G. DeMarco ${ }^{a-e, ~ h ~ L a k s h m i ~ P u l a k a t ~}{ }^{a-d, f-h} \quad$ Melvin R. Hayden ${ }^{b-d, h}$ \\ Tejaswini Joginpally ${ }^{a, c}$ Carlos M. Ferrario ${ }^{i}$ Alan R. Parrish $^{\text {b, e, } g}$ \\ James R. Sowers ${ }^{a-e, ~} h$

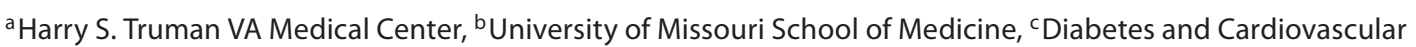 \\ Center, Departments of ${ }^{\mathrm{d}}$ Internal Medicine, ${ }^{\mathrm{e}}$ Medical Pharmacology and Physiology, and ${ }^{\mathrm{f}}$ Nutrition and Exercise \\ Physiology, Divisions of ${ }^{9}$ Nephrology and Hypertension and ${ }^{\text {h}}$ Endocrinology and Metabolism, Columbia, Mo., and \\ 'Wake Forest University, Winston-Salem, N.C., USA
}

\section{Key Words}

Cadherin $\cdot$ Megalin $\cdot \beta-N A G \cdot$ Proteinuria

\begin{abstract}
Background/Aims: The mammalian target of rapamycin (mTOR) is a serine kinase that regulates phosphorylation (p) of its target ribosomal S6 kinase (S6K1), whose activation can lead to glomerular and proximal tubular cell (PTC) injury and associated proteinuria. Increased mTOR/S6K1 signaling regulates signaling pathways that target fibrosis through adherens junctions. Recent data indicate aldosterone signaling through the mineralocorticoid receptor (MR) can activate the mTOR pathway. Further, antagonism of the MR has beneficial effects on proteinuria that occur independent of hemodynamics. Methods: Accordingly, hypertensive transgenic TG(mRen2)27 (Ren2) rats, with elevated serum aldosterone and proteinuria, and age-matched Sprague-Dawley rats were treated with either a low dose ( $1 \mathrm{mg} / \mathrm{kg} / \mathrm{day})$ or a conventional dose (30 mg/kg/day) of spironolactone (MR an-
\end{abstract}

tagonist) or placebo for 3 weeks. Results: Ren2 rats displayed increases in urine levels of the PTC brush border lysosomal enzyme $\mathrm{N}$-acetyl- $\beta$-aminoglycosidase ( $\beta$-NAG) in conjunction with reductions in PTC megalin, the apical membrane adherens protein T-cadherin and basolateral $\alpha$ (E)-catenin, and fibrosis. In concert with these abnormalities, Ren2 renal cortical tissue also displayed increased Ser2448 (p)/activation of mTOR and Thr389 (p)-S6K1 and increased 3-nitrotyrosine (3-NT) content, a marker for peroxynitrite. Low-dose spironolactone had no effect on blood pressure but decreased proteinuria and $\beta$-NAG comparable to a conventional dose of this MR antagonist. Both doses of spironolactone attenuated ultrastructural maladaptive alterations and led to comparable reductions in (p)-mTOR/(p)-S6K1, 3-NT, fibrosis, and increased expression of $\alpha$-(E)-catenin, Tand N-cadherin. Conclusions: Thereby, MR antagonism improves proximal tubule integrity by targeting mTOR/S6K1 signaling and redox status independent of changes in blood pressure.

Copyright $\odot 2011$ S. Karger AG, Basel

\section{KARGER}

Fax +4161306 1234 E-Mail karger@karger.ch www.karger.com
(C) 2011 S. Karger AG, Basel

0250-8095/12/0351-0090\$38.00/0

Accessible online at:

www.karger.com/ajn
Adam Whaley-Connell, DO, MSPH

Harry S. Truman VA Medical Center

800 Hospital Drive, Columbia, MO 65211 (USA)

Tel. +1 5738146000 , ext. 53729

E-Mail whaleyconnella@health.missouri.edu 


\section{Introduction}

Inappropriate activation of mineralocorticoid receptor (MR) signaling pathways, in the absence of salt deprivation, promotes the development of proteinuria and tubulointerstitial fibrosis in the kidney [1-5]. MR-dependent changes in kidney function are thought to largely occur through non-genomic actions including generation of oxidative stress and impairments in metabolic signaling pathways in models of chronic pressure overload $[1,2,5]$. In this context, recent data highlight a role for antagonism of the MR in reducing proteinuria and kidney tubulointerstitial fibrosis, independent of changes in blood pressure $[1,2,5-7]$. It has been suggested that these blood pressure-independent anti-fibrotic effects of MR antagonism are mediated, in part, through correction of abnormalities in metabolic signaling pathways [8].

Elevations in arterial pressure are a critical determinant of maladaptive proximal tubule structure and function over time. However, MR-mediated tubule-interstitial remodeling is also driven by oxidative stress through the enzyme complex NADPH oxidase [9-15]. Redox-sensitive serine kinases such as ribosomal S6 kinase (S6K1), a downstream target of the mammalian target of rapamycin (mTOR) [16], promote tissue remodeling and fibrosis [17-19]. Recent work suggests that activation of mTOR/S6K1 promotes tubulointerstitial remodeling in kidney injury models such as polycystic kidney disease [19-21]. Emerging evidence suggests that strategies targeting reductions in mTOR activity improves fibrosis and proteinuria in rodent models of kidney disease [19].

The adherens junction $\beta$-catenin- $\mathrm{N}$-cadherin complex is critical for maintaining proximal tubular cell (PTC) integrity [17, 22, 23]. Loss of the adherens catenincadherin complex is integral in disruption of PTC adhesion in the early stages of tubulointerstitial fibrosis $[17,22$, 23]. The most extensively studied cadherin is E-cadherin which typically resides in epithelial tissue; however, recent data suggest that $\mathrm{N}$-cadherin is specific to the rat PTCs and binds cytoskeletal components that provide a structural foundation for adherens junctions [22, 24]. Cadherins not only function as static structural components of adherens junctions, but also play an important role in metabolic signaling pathways $[25,26]$. Recent data suggest MR antagonism improves fibrosis through restoration of the adheren $\beta$-catenin- $\mathrm{N}$-cadherin complex [27].

The MR Targets the mTOR Pathway
To these points, we hypothesized that in a rodent model with excess circulating aldosterone the transgenic (TG) (mRen2)27 (Ren2) rat [14, 28], MR blockade would improve proximal tubule structure and function independent of changes in blood pressure. We further posited that this improvement would be associated with reductions in S6K1 phosphorylation (p)/activation and oxidative stress. To evaluate the blood pressure-independent effects of MR antagonism on PTC integrity and proteinuria, we compared a very low, non-pressure-lowering dose of spironolactone (Sp) $[14,29]$ with that of a conventional pressure-lowering dose [30].

\section{Methods}

Animals and Treatments

All animal procedures were approved by the University of Missouri Animal Care and Use Committees and housed in accordance with NIH guidelines. Male Ren2 (R2) and SpragueDawley (SD) littermates (6-7 weeks of age) were assigned to sham treatment (R2-C and SD-C) or randomly assigned to a timed release $\mathrm{Sp}$ pellet designed to deliver either $5 \mathrm{mg}$ (low dose; R2-LSp) or $150 \mathrm{mg}$ of Sp (conventional dose; R2-Sp) via the subcutaneous route for 21 days. The approximate doses for the low dose and conventional dose Sp treatments were 1 and $30 \mathrm{mg} \cdot \mathrm{kg}^{-1} \cdot \mathrm{day}^{-1}$, respectively.

Systolic Blood Pressure (SBP) and Albuminuria

Restraint conditioning was initiated before blood pressure measurements were performed as previously described [14, 30]. SBP was measured in triplicate on separate occasions throughout the day using the tail-cuff method (Harvard Systems, Student Oscillometric Recorder) prior to initiation of treatment and on days 19 or 20 and prior to sacrifice at 21 days. Urine albumin and creatinine were determined as previously described [14, 30].

\section{Western Blot Analysis}

SDS-PAGE was performed on kidney homogenates. Samples (40 $\mu \mathrm{g} /$ lane) were separated and transferred to PVDF membranes. Blots were blocked with 5\% BSA for $3 \mathrm{~h}$ and then washed briefly. Blots were incubated overnight at $4^{\circ} \mathrm{C}$ with the following primary antibodies: (p)-mTOR, (p)-S6K1, N-cadherin, and $\alpha(E)$ catenin (1:1,000 dilution; BD, Inc.). After rinsing, blots were incubated with horseradish peroxidase-conjugated secondary antibodies for $1 \mathrm{~h}$ at room temperature. The bands were visualized by chemiluminescence, and images were recorded using a BioRad image analysis system. The quantified proteins were normalized to the density of total protein for each sample, as determined by amido black, and performed using Quantity One software.

Immunohistochemical Quantification

Four micrometers of paraffin-embedded sections of kidney were deparaffinized in CitriSolv, rehydrated in ethanol series and Hepes buffer $[30,31]$. Epitopes were retrieved (antigen retrieval) in citrate buffer $\mathrm{pH} 6$ for $25 \mathrm{~min}$ at $95^{\circ} \mathrm{C}$. Slides were then 
immediately transferred into a humidity chamber. Non-specific binding sites were blocked (5\% BSA, 5\% serum of the animals in which secondary antibodies were generated, $0.01 \%$ sodium azide) at room temperature for $4 \mathrm{~h}$. After a gentle wash with Hepes, the sections were incubated with primary antibodies, 1:50 goat polyclonal (p)2448-mTOR, (p)389-S6K1, megalin, Tcadherin and 1:100 Nox2. All primary antibodies were diluted in tenfold diluted blocker. The sections were washed $(3 \times 15$ min) and incubated with 1:300 Alexa flour 647 donkey antimouse, rabbit or goat corresponding with the animals that the primary antibodies were generated for $4 \mathrm{~h}$. The slides were then washed $(3 \times 15 \mathrm{~min})$ and sections mounted with Mowiol and stored in light-tight slide boxes at $4^{\circ} \mathrm{C}$ until assessment using a bi-photon confocal microscope (Zeiss LSM, 510 MLO; Zeiss, Thornwood, N.Y., USA). 1,024 × 1,024 pixel images were captured with an LSM imaging system under the same microscope and computer settings for all animals of four groups in each experiment. The signal intensities were measured and analyzed in similar-sized regions with MetaView.

\section{Ultrastructural Analysis with Transmission Electron}

Microscopy (TEM) Methods

Kidney cortical tissue was thinly sliced and placed immediately in primary TEM fixative as previously described [14, 31]. Sections $(85 \mathrm{~nm})$ were stained with $5 \%$ uranyl acetate and Sato's triple-lead stain and a TEM (model JEM 1400; Joel, Ltd, Tokyo, Japan) was utilized. To maintain uniformity, we examined S-1 segments of the proximal tubules ( $n=4$ per group) with identifiable microvilli that were immediately adjacent to glomeruli.

\section{Statistical Analysis}

All values are expressed as mean \pm SE. Statistical analyses were performed using ANOVA with Tukey's post-hoc test for all other outcomes and test for proximal tubule measures (Sigma Stat 3.1; Systat Software Inc., Chicago, Ill., USA).

\section{Results}

\section{Spironolactone Treatment Improves PTC Injury}

We recently reported [30] significant increases in circulating aldosterone and SBP in young (9-10 weeks of age) Ren 2 rats compared to SD littermate controls. In the same investigation, low-dose $\mathrm{Sp}$ did not reduce SBP which decreased significantly with the conventional dose of Sp [14]. SBP is an important factor in mediating progressive kidney disease as measured by proteinuria. In this context, there were increases in proteinuria in Ren 2 compared to SD controls, findings that were prevented over 3 weeks of treatment to a similar extent with both the low dose as well as a conventional dose of $\mathrm{Sp}$ (fig. 1a).

PTC injury is characterized by release of enzymes such as $\mathrm{N}$-acetyl- $\beta$-aminoglycosidase ( $\beta-\mathrm{NAG}$ ), a lysosomal enzyme marker specific to the endosomal brush border. Similar to SBP and proteinuria in Ren 2 rats, there were increases in $\beta$-NAG compared to SD controls. Comparable to our observed reductions in proteinuria with both doses of $S p$, there were similar reductions in $\beta$-NAG with both the low and conventional dose of Sp (fig. 1b).

Injury to the PTC is associated with loss of the brush border endocytotic receptor megalin that is responsible for PTC reabsorption of protein. To this point, the increases in proteinuria and $\beta-\mathrm{NAG}$ in Ren 2 controls was associated with a parallel reduction in megalin immunostaining in Ren2 renal cortical tissue (fig. 1c). Treatment with both doses of Sp improved megalin immunostaining in the Ren2 (fig. 1c). Ultrastructural analysis of the PTC endosomal region with TEM corroborated the megalin findings.

Representative images (fig. 1d) depict reductions in the number of electron-dense lysosomes (arrows) in the Ren2 compared to SD controls, restored with both doses of Sp.

\section{Spironolactone Treatment Improves PTC Oxidative Stress}

PTC injury is, in part, due to generation of excess reactive oxygen species (ROS) $[1,4,5]$ by the enzyme complex NADPH oxidase [32]. PTCs have a higher endogenous capacity for NOX-dependent ROS generation than glomerular cells. In this context, there were greater increases in the NADPH oxidase subunit Nox2 and 3-nitrotyrosine (3-NT), a marker of peroxynitrite (ONOO-) formation, in the PTC Ren 2 compared to SD renal tissue (fig. 2a, b). Treatment with both doses of Sp reduced Ren2 PTC Nox2 and 3-NT content to a similar extent.

Fig. 1. MR receptor antagonism with Sp improves PTC injury in the TG Ren2 (R2) rat. a Proteinuria. b Urine $\beta$-NAG. c Representative images of semiquantitative immunohistochemical analysis of megalin with corresponding measures to the right. Values represented as mean \pm SEM. ${ }^{*} \mathrm{p}<0.05$ when Ren 2 controls (R2-C) are compared to SD controls (SD-C); ${ }^{*} \mathrm{p}<0.05$ when Sp treated Ren2s at low dose (R2-LSp) or conventional dose (R2-Sp) are compared to R2-C. $\times 800$. Scale bar $=50 \mu \mathrm{m}$. d Ultrastructural analysis of the proximal tubule utilizing TEM and noted electron-dense lysosomes (open arrows). $\times 800$. Scale bar $=2 \mu \mathrm{m}$. 

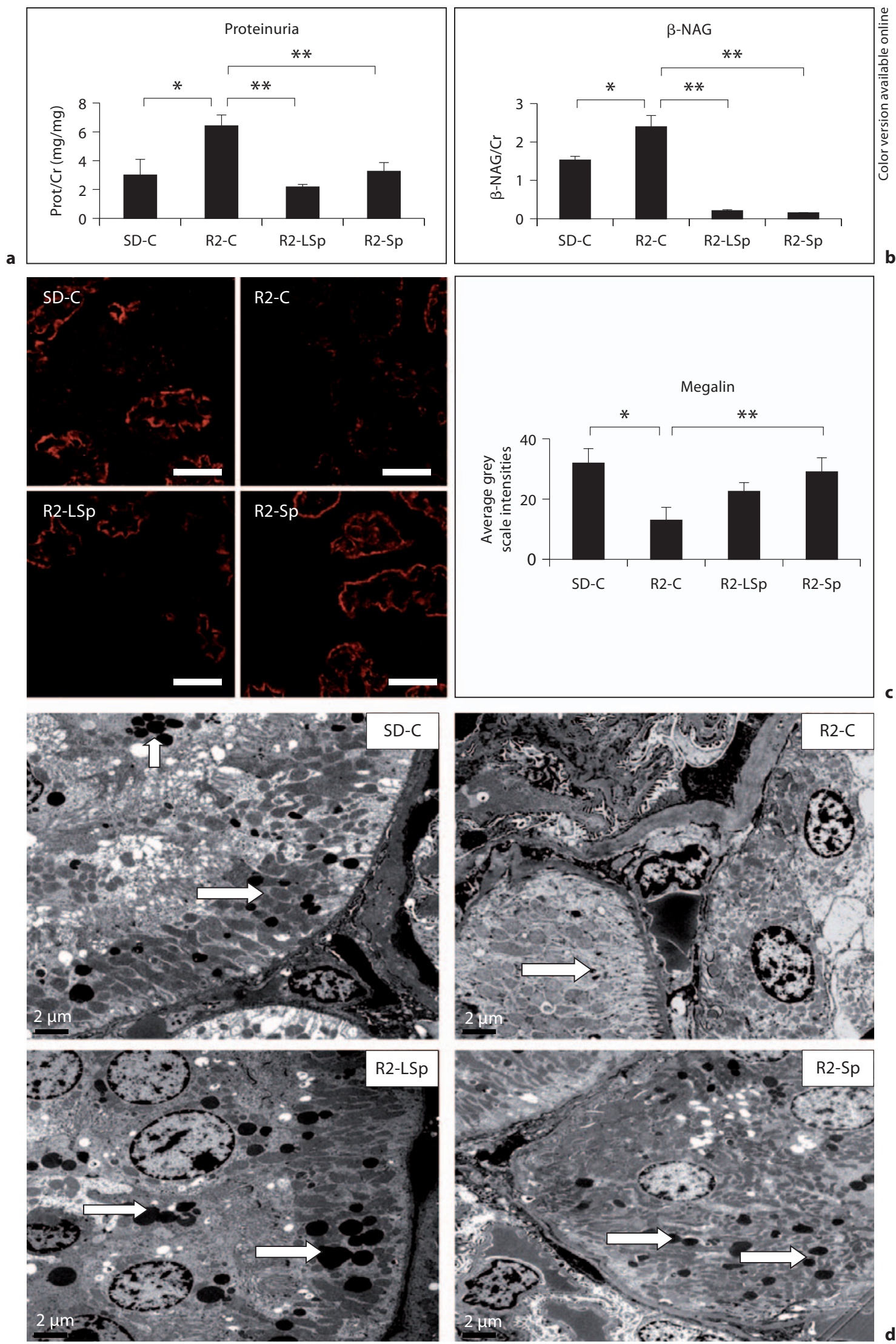

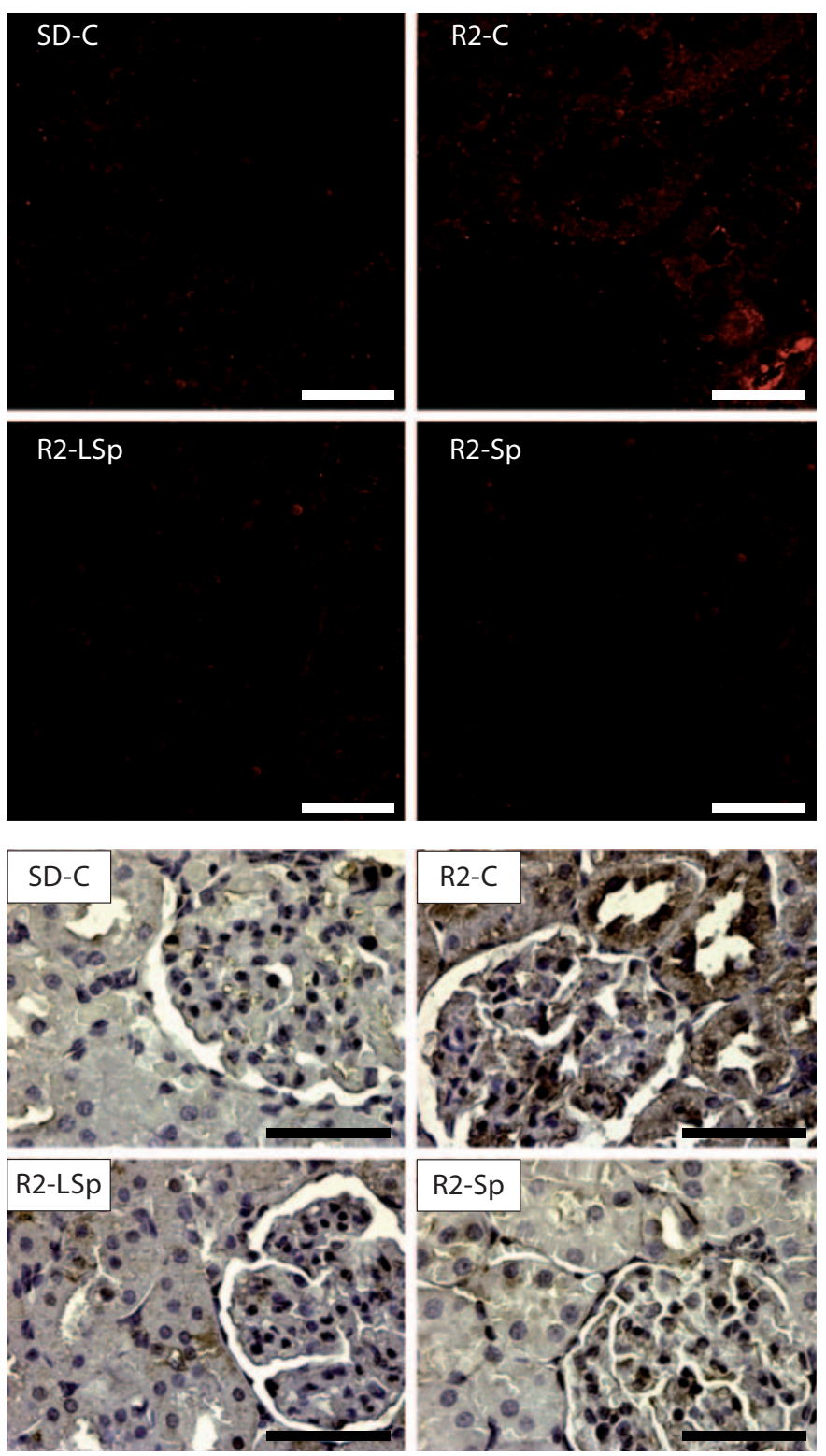

Fig. 2. MR antagonism with $\mathrm{Sp}$ improves proximal tubule oxidative stress in the TG Ren2 (R2) rat. a Depicts representative images of semiquantitative immunohistochemistry analysis of NADPH oxidase (Nox) subunit Nox 2 with corresponding measures of intensity to the right. b Representative images of 3-NT content, a marker of peroxynitrite formation $\left(\mathrm{ONOO}^{-}\right)$, with cor-

Spironolactone Treatment Reduces Activation/(p) of mTOR/S6K1

Recent data highlight that the mTOR/S6K1 pathway is redox-sensitive [16]. Contemporaneous with the increases in Nox 2 and 3-NT in Ren 2 cortical tissue (fig. 2), there were increased levels of Ser2448 p-mTOR and Thr389 p-
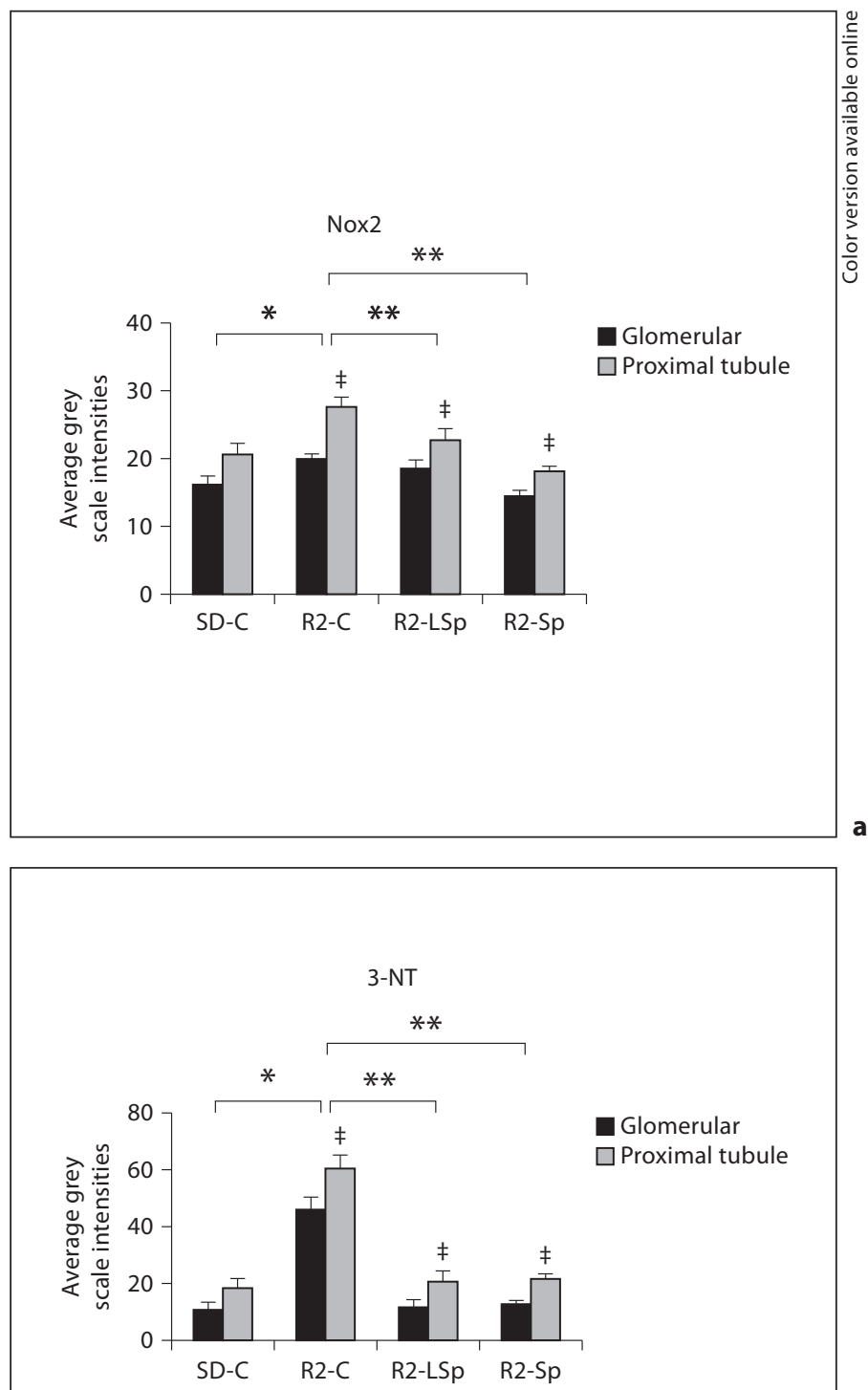

Glomerular

$\square$ Proximal tubule

responding measures to the right. Values represented as mean \pm SEM. ${ }^{*} \mathrm{p}<0.05$ when Ren 2 controls (R2-C) are compared to SD controls (SD-C); ${ }^{* *} \mathrm{p}<0.05$ when Sp treated Ren2s at low dose (R2-LSp) or conventional dose (R2-Sp) are compared to R2-C; ${ }^{\ddagger} \mathrm{p}<0.05$ when proximal tubule intensities are compared to glomerular intensities. Scale bar $=50 \mu \mathrm{m}$.

S6K1 on Western blot analysis and immunostaining of the PTC (fig. 3a, b). The enhanced (p) of mTOR and S6K1 were significantly decreased with low-dose and conventional-dose $\mathrm{Sp}$ treatment. However, the conventional dose had a somewhat greater impact in reducing $\mathrm{p}-\mathrm{S} 6 \mathrm{~K} 1$ compared to the low dose. 
Fig. 3. MR antagonism with $\mathrm{Sp}$ improves proximal tubule mTOR signaling in the TG Ren2 (R2) rat. a Western blot analysis of serine (Ser) 2448 phosphorylation (p) of mTOR and threonine (Thr) 389 phosphorylation ( $p$ ) of S6K1. b Representative images of semiquantitative immunohistochemical analysis of proximal tubule Ser2448 (p)-mTOR. Values represented as mean \pm SEM. ${ }^{*} \mathrm{p}<0.05$ when Ren 2 controls (R2-C) are compared to SD controls (SD-C); ${ }^{* *} \mathrm{p}<0.05$ when Sp treated Ren $2 \mathrm{~s}$ at low dose (R2-LSp) or conventional dose (R2-Sp) are compared to R2-C; ${ }^{*} \mathrm{p}<0.05$ when R2-Sp is compared to R2-LSp. Scale bar $=50 \mu \mathrm{m}$.
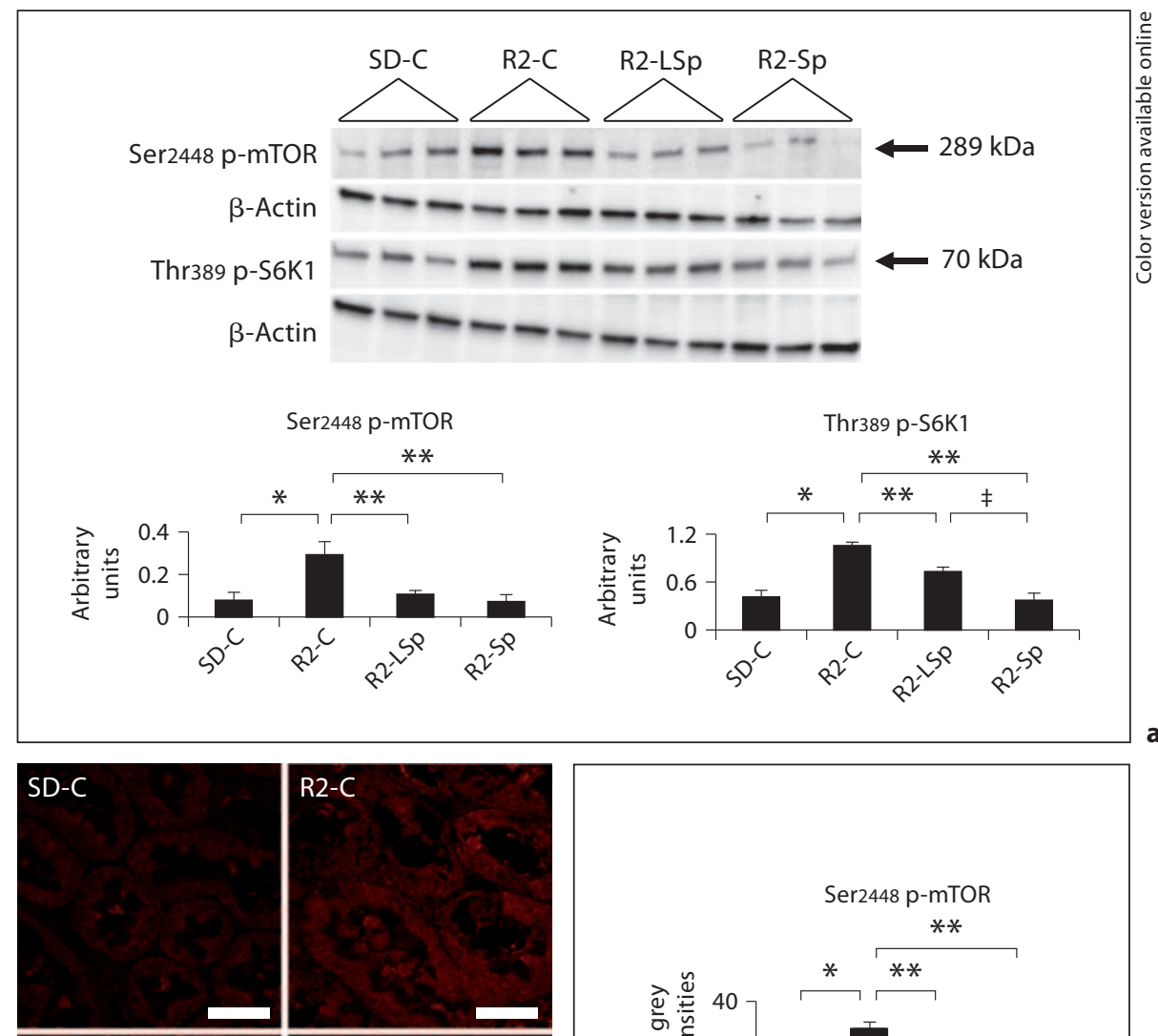

\section{R2-LSp}

\section{R2-SP}

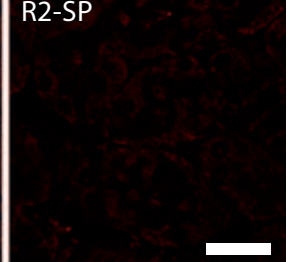

Spironolactone Treatment Improves PTC Adherens

Proteins

The mTOR/S6K1 pathway has specific effects on adhesion through translational regulation of adherens junctions. Recent work suggests that adhesion molecules such as cadherins have a specific role in cell signaling signal recognition as well as PTC structural integrity $[24,25]$. A recently identified non-classic T-cadherin has been localized to the apical surface of PTCs in lipid rafts that functions in cell signal recognition $[33,34]$. In this context, T-cadherin localized to PTCs to a greater extent than to the glomerulus on immunostaining (fig. 4a). There were diminished levels of T-cadherin in Ren 2 renal tissue, but levels of this adhesion molecule were normalized with both doses of Sp.

The MR Targets the mTOR Pathway
Loss of PTC adhesion and subsequent tubulointerstitial fibrosis occurs through disassociation of catenins followed by down-regulation of cadherin [35]. Ren2 renal tissue displayed reductions compared to $\mathrm{SD}$ controls in $\alpha$-(E)-catenin but not $\mathrm{N}$-cadherin (fig. $4 \mathrm{~b})$. Both doses of Sp led to increases in $\alpha-(\mathrm{E})$-catenin and $\mathrm{N}$-cadherin, whereas a conventional dose of an MR antagonist significantly improved $\alpha-(\mathrm{E})$-catenin. Commensurate with abnormalities in the adherens protein catenin-cadherin complex, on ultrastructural analysis Ren2 PTCs displayed qualitative reductions in length $(\sim 50-100 \mathrm{~nm}$; $\mathrm{n}=3$ ) of adherens junctions compared to SD controls $(\sim 700-1,400 \mathrm{~nm} ; \mathrm{n}=3)$ (fig. 4c). There were increases in length of adherens junctions $(300-400 \mathrm{~nm} ; \mathrm{n}=3$ each) in Ren2 PTCs with both doses of Sp. 


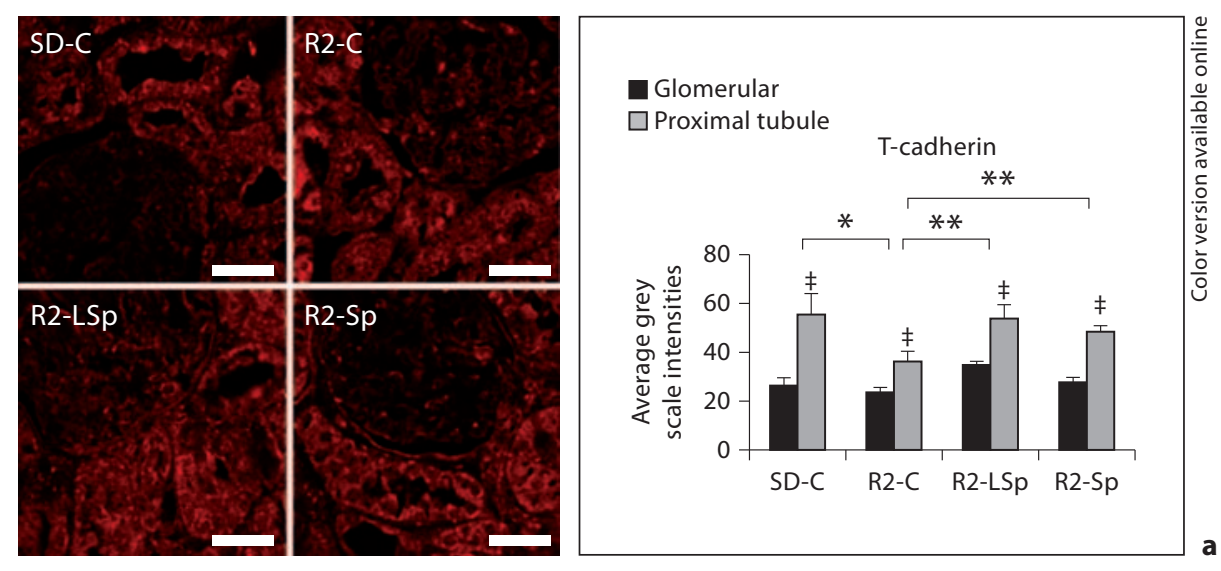

Fig. 4. MR antagonism with $\mathrm{Sp}$ improves proximal tubule adherens proteins in the TG Ren2 (R2) rat. a Representative images of semiquantitative immunohistochemical analysis of T-cadherin. Values represented as mean \pm SEM. ${ }^{\ddagger} \mathrm{p}<0.05$ when proximal tubule is compared to glomerular staining. Scale bar $=50 \mu \mathrm{m}$. b Western blot analysis of adherens junction proteins. ${ }^{*} \mathrm{p}<0.05$ when Ren 2 controls (R2C) are compared to SD controls (SD-C); ** $\mathrm{p}<0.05$ when Sp treated Ren2s at low dose (R2-LSp) or conventional dose (R2$\mathrm{Sp}$ ) are compared to R2-C. c Ultrastructural analysis of the proximal tubule cellcell adhesion utilizing TEM and noted electron-dense adherens junctions (open arrows). $\times 20,000$. Bar $=100 \mathrm{~nm}$.
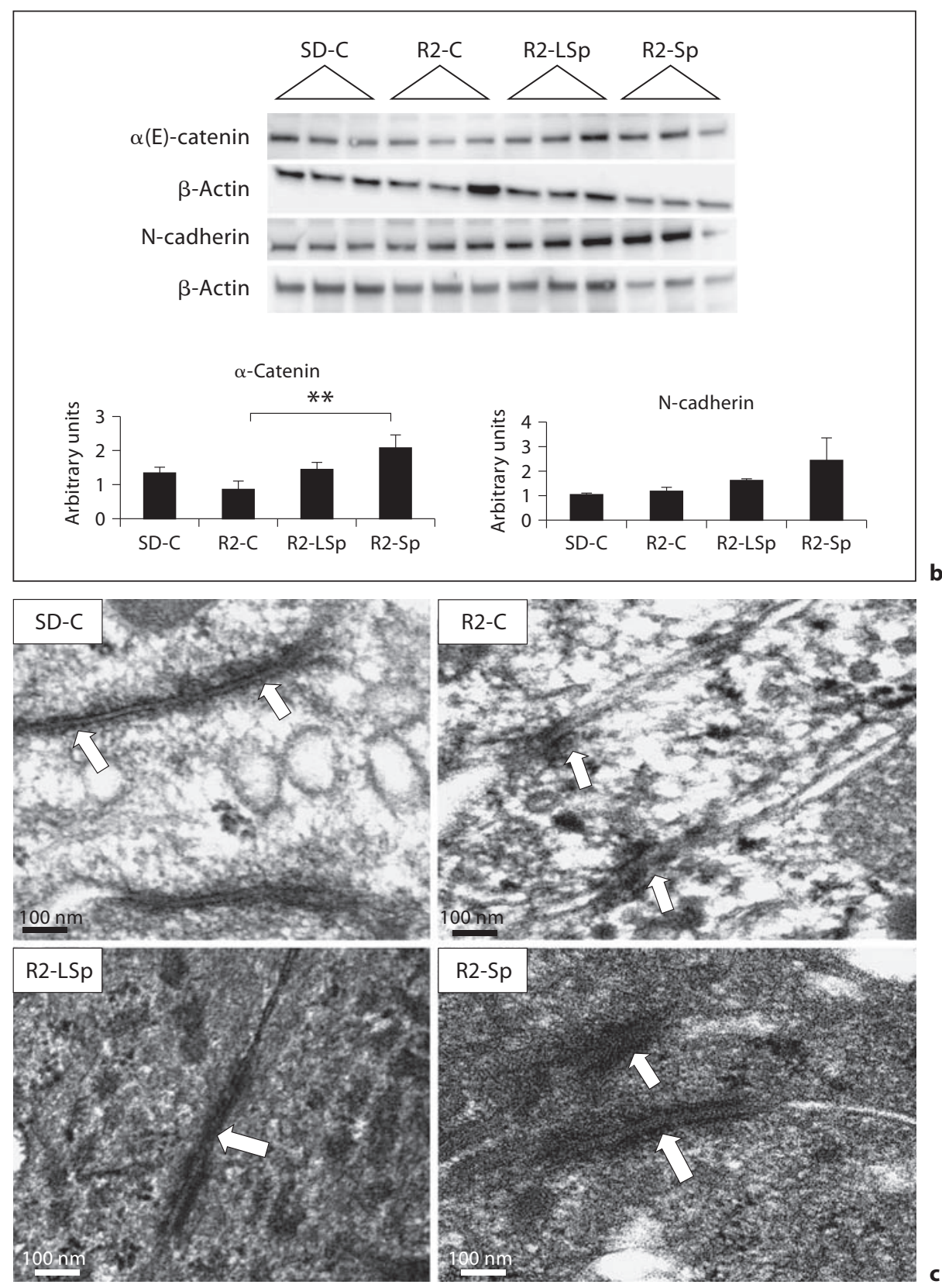

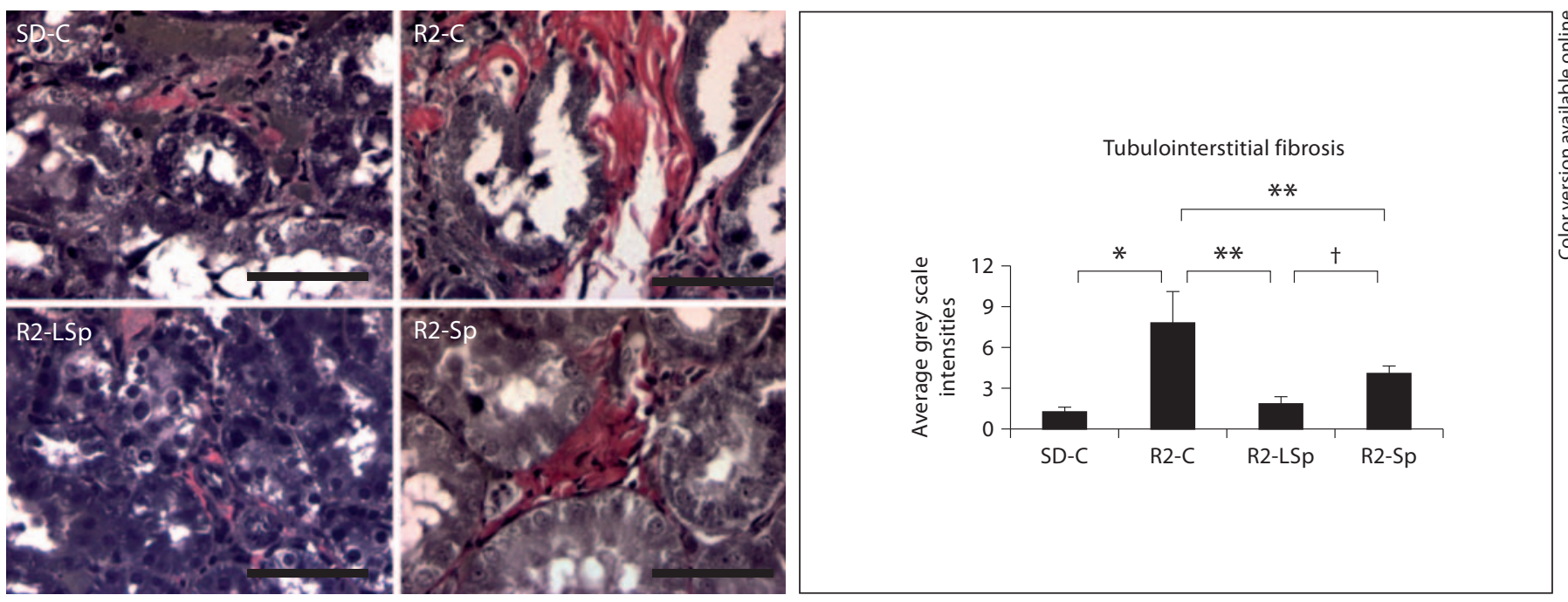

Fig. 5. MR antagonism with Sp improves tubulointerstitial fibrosis in the TG Ren2 (R2) rat. Verhoeff-Van Gieson stain for elastin and collagen with measures of tubulointerstitial fibrosis to the right. Values represented as mean \pm SEM. ${ }^{*} \mathrm{p}<0.05$ when Ren 2 controls (R2-C) are compared to SD controls (SD-C); ${ }^{* *}$ p $<0.05$ when Sp treated Ren2s at low dose (R2-LSp) or conventional dose (R2-Sp) are compared to R2-C; ${ }^{\dagger} \mathrm{p}<0.05$ when lowdose Sp compared to conventional-dose Sp in the R2. Scale bar $=50 \mu \mathrm{m}$.

In this context, the Ren2 displayed increases in tubulointerstitial fibrosis compared to SD controls, findings that were attenuated with both doses of Sp (fig. 5). Further, despite not reducing SBP, the low dose improved fibrosis to a greater extent than conventional dose of Sp.

\section{Discussion}

Results of this investigation support a role in PTC injury for MR signaling through the redox-sensitive mTOR/ S6K1 pathway in a young TG rat model with elevated plasma levels of aldosterone. Previous work in the Ren2, as well as the Cyp1a1-Ren2, suggests that MR antagonism leads to improvements in glomerular damage and proteinuria without reductions in SBP $[7,30]$. Recent work from our group and others suggest that at early stages of kidney injury there are urine protein contributions derived from either impairments in megalin-mediated endocytosis or the degradation process in PTCs $[31,36]$. A key role for PTC injury was evidenced by increases in $\beta$ NAG coupled with reductions in megalin and ultrastructural evidence of loss of PTC integrity. PTC injury is further evident in Ren 2 kidneys by loss of the apically ex- pressed, glycosyl-phosphatidyl-inositol (GPI)-anchored T-cadherin and disruption in the $\alpha-(\mathrm{E})$-catenin-N-cadherin complex with tubulointerstitial fibrosis. These structural abnormalities occurred in concert with activation of oxidative stress (Nox2 and 3-NT content) and enhanced (p) of mTOR/S6K1. The finding that low-dose and conventional Sp treatment comparably reduced oxidative stress and redox-sensitive mTOR/S6K1 activation and improved PTC structural integrity supports the notion of a direct deleterious effect of MR-mediated activation of the mTOR/S6K1 pathway on PTC structure and function independent of increased blood pressure in this TG model.

Renal cortical tissue from the TG Ren2 displayed increased Nox 2 and 3-NT content in concert with associated increases in (p)/activation of mTOR and S6K1 in the PTC. Recent work highlights a very specific role for mTOR as a redox-sensitive kinase in the PTC, whereby increases in Ser2448 (p) lead to downstream activation/ (p) of S6K1 at Thr389 in eliciting fibrotic pathways under agonist conditions $[16,37,38]$. It was recently reported that treatment of cultured PTCs with oxidizing compounds that induce disulfide bonds between the thiol groups of cysteines in proteins promoted Thr389 (p) of S6K1 [37]. This promotion of S6K1 (p) was mediated 
through mTOR kinase activation, suggesting the redoxsensitive nature of the kinase (p)/activation of S6K1 as observed in the current investigation.

The regulatory role of the mTOR/S6K1 pathway in the kidney has been shown to occur through progressive processes involving PTC hypertrophy, atrophy and then development of fibrosis through disruption of the catenin-cadherin complex [20,39]. Under basal steady-state conditions, PTCs are attached to each other through specialized junctional complexes (adherens junctions) that include molecules such as cadherins. During albumininduced injury, PTCs and other epithelial cells lose polarity and the mechanisms of adhesion through loss of cadherins [40]. A recently identified cadherin, T-cadherin, also known as $\mathrm{H}$-cadherin (CDH13), is characterized by the lack of a transmembrane region, cytoplasmatic tail, and a missing conserved cell-adhesion recognition site. This suggests the cellular properties of T-cadherin in the kidney are different than the classical cadherins such as $\mathrm{E}$ - and $\mathrm{N}$-cadherin. To this point, the $\mathrm{C}$-terminus of $\mathrm{T}$ cadherin is bound and localizes on defined domains of the plasma membrane, e.g. lipid rafts. Lipid rafts, in turn, are of functional importance for the polarization of epithelial cells, mainly by sorting out apical membrane proteins such as megalin.

Our finding that proximal tubule staining of T-cadherin is reduced in the Ren2, in parallel with decreased megalin expression, suggests a role for T-cadherin in PTC function. Current observations that both doses of Sp increased T-cadherin suggest a potential role for the MR in targeting T-cadherin in the proximal tubule. In this context, our data extend findings from PTC culture models in recent studies $[7,17,20,40,41]$ to an in vivo model (e.g. the Ren2), where MR-dependent signaling through mTOR/S6K1 reduces megalin expression that occur in conjunction with loss of T-cadherin.

Current data is consistent with the notion that the classical cadherin/catenin complex is a target of the MR. Our finding that $\alpha$-(E)-catenin but not $\mathrm{N}$-cadherin was reduced in the Ren 2 compared to the SD may represent a time-dependent mechanism in disassociation of the catenin-cadherin complex [35], and this needs to be explored in more detail in the future. Data from the heart of TG mice developing myocardial fibrosis suggest that aldosterone signaling through the MR has a specific role in eliciting disruptions in the $\beta$-catenin- $\mathrm{N}$-cadherin complex and fibrosis [37]. However, there is little extant data demonstrating that changes in mTOR/S6K1 (p) contribute to changes in cadherin expression in rodent models of MR activation. Our finding that a low, non-pressor- related dose of $\mathrm{Sp}$ improved tubulointerstitial fibrosis along with cadherin/catenin complex integrity supports a novel role for the MR in PTCs. As disruption of catenincadherin complex may initiate tubulointerstitial fibrosis [35], the reduction in $\alpha$-(E)-catenin coupled with ultrastructural observations suggest that fibrosis was initiated by disruptions in the $\alpha$-(E)-catenin- $\mathrm{N}$-cadherin complex in this TG model.

Thus, our data highlight a novel role for accentuated MR signaling in the genesis of proximal tubule-related proteinuria through the redox-sensitive (p) of mTOR/ S6K1 leading to downstream disruption of the catenincadherin complex and tubulointerstitial fibrosis. While our study did not include a direct inhibitor of Ser2448 phosphorylation of mTOR such as rapamycin or an antioxidant, to our knowledge the current data are the first to explore the blood pressure-independent actions of the MR in promotion of PTC and tubulointerstitial injury in a rodent model with elevated circulating aldosterone levels.

\section{Acknowledgments}

The authors would like to acknowledge the support of the Electron Microscopy Core and Charles Wiedmeyer, DVM, PhD. They also acknowledge the technical contributions of Rebecca Schneider, Nathan Rehmer, and Mona Garro as well as students Safwan Hyder and Bennett Krueger. The authors thank Brenda Hunter for her assistance in preparing the manuscript.

This research was supported by the NIH R01 HL-73101 and NIH R01 HL-107910 to J.R.S., and R-03 AG040638 to A.T.W.-C. There was also support from the Veterans Affairs Merit System (0018) for J.R.S. as well as CDA-2 to A.T.W.-C. and the ASN-ASP Junior Development Grant in Geriatric Nephrology to A.T.W.-C. supported by a T. Franklin Williams Scholarship Award. Funding was provided by Atlantic Philanthropies, Inc., the John A. Hartford Foundation, the Association of Specialty Professors, and the American Society of Nephrology.

\section{Disclosure Statement}

The authors have no conflicts of interest to disclose. 


\section{References}

$>1$ Bertocchio JP, Warnock DG, Jaisser F: Mineralocorticoid receptor activation and blockade: an emerging paradigm in chronic kidney disease. Kidney Int 2011;79:10511060.

\2 Brown NJ, Nakamura S, Ma L, Nakamura I, Donnert E, Freeman M, Vaughan DE, Fogo AB: Aldosterone modulates plasminogen activator inhibitor-1 and glomerulosclerosis in vivo. Kidney Int 2000;58:1219-1227.

$\checkmark 3$ Cooper SA, Whaley-Connell A, Habibi J, Wei Y, Lastra G, Manrique C, Stas S, Sowers JR: Renin-angiotensin-aldosterone system and oxidative stress in cardiovascular insulin resistance. Am J Physiol Heart Circ Physiol 2007:293:H2009-H2023.

$\checkmark 4$ Funder JW: The non-genomic actions of aldosterone. Endocr Rev 2005;26:313-321.

$\checkmark 5$ Kawarazaki H, Ando K, Fujita M, Matsui H, Nagae A, Muraoka K, Kawarasaki C, Fujita T: Mineralocorticoid receptor activation: a major contributor to salt-induced renal injury and hypertension in young rats. Am J Physiol Renal Physiol 2011;300:F1402F1409.

-6 Nagata K, Obata K, Xu J, Ichihara S, Noda A, Kimata H, Kato T, Izawa H, Murohara T, Yokota M: Mineralocorticoid receptor antagonism attenuates cardiac hypertrophy and failure in low-aldosterone hypertensive rats. Hypertension 2006;47:656-664.

7 Ortiz RM, Graciano ML, Mullins JJ, Mitchell $\mathrm{KD}$ : Aldosterone receptor antagonism alleviates proteinuria, but not malignant hypertension, in Cypla1-Ren2 transgenic rats. Am J Physiol Renal Physiol 2007;293:F1584F1591.

$>8$ Boldyreff B, Wehling M: Aldosterone: refreshing a slow hormone by swift action. Physiology 2004;19:97-100.

9 Callera GE, Touyz RM, Tostes RC, Yogi A, He Y, Malkinson S, Schiffrin EL: Aldosterone activates vascular $\mathrm{p} 38 \mathrm{MAP}$ kinase and NADPH oxidase via c-Src. Hypertension 2005;45:773-779.

- 10 Johar S, Cave AC, Narayanapanicker A, Grieve DJ, Shah AM: Aldosterone mediates angiotensin II-induced interstitial cardiac fibrosis via a Nox2-containing NADPH oxidase. FASEB J 2006;20:1546-1548.

-11 Kobayashi N, Yoshida K, Nakano S, Ohno T, Honda T, Tsubokou Y, Matsuoka H: Cardioprotective mechanisms of eplerenone on cardiac performance and remodeling in failing rat hearts. Hypertension 2006;47:671679 .

-12 Miyata K, Rahman M, Shokoji T, Nagai Y, Zhang GX, Sun GP, Kimura S, Yukimura T, Kiyomoto H, Kohno M, Abe Y, Nishiyama A: Aldosterone stimulates reactive oxygen species production through activation of NADPH oxidase in rat mesangial cells. J Am Soc Nephrol 2005;16: 2906-2912.
13 Montezano AC, Callera GE, Yogi A, He Y, Tostes RC, He G, Schiffrin EL, Touyz RM: Aldosterone and angiotensin II synergistically stimulate migration in vascular smooth muscle cells through c-Src-regulated redox-sensitive RhoA pathways. Arterioscler Thromb Vasc Biol 2008;28:1511-1518.

14 Whaley-Connell A, Habibi J, Wei Y, Gutweiler A, Jellison J, Wiedmeyer CE, Ferrario CM, Sowers JR: Mineralocorticoid receptor antagonism attenuates glomerular filtration barrier remodeling in the transgenic Ren2 rat. Am J Physiol Renal Physiol 2009; 296:F1013-F1022.

15 Tabet F, Schiffrin EL, Callera GE, He Y, Yao G, Ostman A, Kappert K, Tonks NK, Touyz RM: Redox-sensitive signaling by angiotensin II involves oxidative inactivation and blunted phosphorylation of protein tyrosine phosphatase SHP-2 in vascular smooth muscle cells from SHR. Circ Res 2008;103:149158.

16 Yalcin S, Marinkovic D, Mungamuri SK Zhang X, Tong W, Sellers R, Ghaffari S: ROSmediated amplification of AKT/mTOR signalling pathway leads to myeloproliferative syndrome in Foxo3 ${ }^{-/-}$mice. EMBO J 2010: 29: 4118-4131.

17 Bieri M, Oroszlan M, Zuppinger C, Mohacsi PJ: Biosynthesis and expression of VE-cadherin is regulated by the $\mathrm{PI} 3 \mathrm{~K} / \mathrm{mTOR}$ signaling pathway. Mol Immunol 2009;46:866872 .

18 Chen JK, Chen J, Thomas G, Kozma SC, Harris RC: S6 kinase-1 knockout inhibits uninephrectomy- or diabetes-induced renal hypertrophy. Am J Physiol Renal Physiol 2009 297:F585-F593.

19 Nakagawa S, Masuda S, Nishihara K, Inui K: mTOR inhibitor everolimus ameliorates progressive tubular dysfunction in chronic renal failure rats. Biochem Pharmacol 2010; 79:67-76.

20 Bonegio RG, Fuhro R, Wang Z, Valeri CR, Andry C, Salant DJ, Lieberthal W: Rapamycin ameliorates proteinuria-associated tubulointerstitial inflammation and fibrosis in experimental membranous nephropathy. J Am Soc Nephrol 2005; 16:2063-2072.

-21 Lieberthal W, Levine IS: The role of the mammalian target of rapamycin (mTOR) in renal disease. J Am Soc Nephrol 2009;20: 2493-502.

-22 Jung KY, Dean D, Jiang J, Gaylor S, Griffith WH, Burghardt RC, Parrish AR: Loss of Ncadherin and $\alpha$-catenin in the proximal tubules of aging male Fischer 344 rats. Mech Ageing Dev 2004;125:445-453.

23 Okada H, Kalluri R: Cellular and molecular pathways that lead to progression and regression of renal fibrogenesis. Curr Mol Med 2005;5:467-474
24 Parrish AR, Catania JM, Orozco J, Gandolfi AJ: Chemically induced oxidative stress disrupts the E-cadherin/catenin cell adhesion complex. Toxicol Sci 1999;51:80-86.

$\checkmark 25$ Chu YS, Thomas WA, Eder O, Pincet F, Perez E, Thiery JP, Dufour S: Force measurements in E-cadherin-mediated cell doublets reveal rapid adhesion strengthened by actin cytoskeleton remodeling through $\mathrm{Rac}$ and Cdc42. J Cell Biol 2004;167:1183-1194.

26 Lee JM, Dedhar S, Kalluri R, Thompson EW: The epithelial-mesenchymal transition: new insights in signalling, development, and disease. J Cell Biol 2006;172: 973-981.

27 Tsybouleva N, Zhang L, Chen S, Patel R, Lutucuta S, Nemoto S, DeFreitas G, Entman M, Carabello BA, Roberts R, Marian AJ: Aldosterone, through novel signaling proteins, is a fundamental molecular bridge between the genetic defect and the cardiac phenotype of hypertrophic cardiomyopathy. Circulation 2004;109:1284-1291.

28 Sander M, Bader M, Djavidani B, MaserGluth C, Vecsei P, Mullins J, Ganten D, Peters J: The role of the adrenal gland in hypertensive transgenic rat TGR(mREN2)27. Endocrinology 1992;131:807-814.

29 Wei Y, Whaley-Connell AT, Habibi J, Rehmer J, Rehmer N, Patel K, Hayden M, DeMarco V, Ferrario CM, Ibdah JA, Sowers JR: Mineralocorticoid receptor blockade attenuates vascular apoptosis and injury. Hypertension 2009;53:158-165.

30 Habibi J, Demarco VG, Ma L, Pulakat L, Rainey WE, Whaley-Connell AT, Sowers JR: Mineralocorticoid receptor blockade improves diastolic function independent of blood pressure reduction in a transgenic model of RAAS overexpression. Am J Physiol Heart Circ Physiol 2011;300:H1484H1491.

-31 Habibi J, Hayden MR, Sowers JR, Pulakat L, Tilmon RD, Manrique C, Lastra G, Demarco VG, Whaley-Connell A: Nebivolol attenuates redox-sensitive glomerular and tubular mediated proteinuria in obese rats. Endocrinology 2011;152:659-668.

32 Whaley-Connell AT, Chowdhury NA, Hayden MR, Stump CS, Habibi J, Wiedmeyer CE, Gallagher PE, Tallant EA, Cooper SA, Link CD, Ferrario C, Sowers JR: Oxidative stress and glomerular filtration barrier injury: role of the renin-angiotensin system in the Ren 2 transgenic rat. Am J Physiol Renal Physiol 2006;291:F1308-F1314.

-33 Arnemann J, Sultani O, Hasgün D, Coerdt W: T-/H-cadherin (CDH13): a new marker for differentiating podocytes. Virchows Arch 2006;448:160-164.

34 Carter WB, Niu G, Ward MD, Small G, Hahn JE, Muffly BJ: Mechanisms of HER2-induced endothelial cell retraction. Ann Surg Oncol 2007;14:2971-2978. 
-35 Koller E, Ranscht B: Differential targeting of $\mathrm{T}$ - and $\mathrm{N}$-cadherin in polarized epithelial cells. J Biol Chem 1996;271:30061-30067.

-36 Russo LM, Sandoval RM, Campos SB, Molitoris BA, Comper WD, Brown D: Impaired tubular uptake explains albuminuria in early diabetic nephropathy. J Am Soc Nephrol 2010;20:489-494.

>37 Sarbassov DD, Sabatini DM: Redox regulation of the nutrient-sensitive raptor-mTOR pathway and complex. J Biol Chem 2005;280: 39505-39509.
38 Xu G, Liu A, Liu X: Aldosterone induces collagen synthesis via activation of extracellular signal-regulated kinase 1 and 2 in renal proximal tubules. Nephrology 2008;13:694701.

39 Whaley-Connell A, Habibi J, Panfili Z, Hayden MR, Bagree S, Nistala R, Hyder S, Krueger B, Demarco V, Pulakat L, Ferrario CM, Parrish A, Sowers JR: Angiotensin II activation of mTOR results in tubulointerstitial fibrosis through loss of $\mathrm{N}$-cadherin. Am J Nephrol 2011;34:115-125.
40 Lee JY, Chang JW, Yang WS, Kim SB, Park SK, Park JS, Lee SK: Albumin-induced epithelial-mesenchymal transition and ER stress are regulated through a common ROSc-Src kinase-mTOR pathway: effect of imatinib mesylate. Am J Physiol Renal Physiol 2011;300:F1214-F1222.

41 Oroszlán M, Bieri M, Ligeti N, Farkas A, Meier B, Marti HP, Mohacsi P: Sirolimus and everolimus reduce albumin endocytosis in proximal tubule cells via an angiotensin IIdependent pathway. Transpl Immunol 2010; 23:125-132. 